\title{
Performance Analysis and Comparison between Uplink and Downlink Techniques in LTE Network
}

\author{
Puja Julaha \\ Department of Computer \& Science Engineering \\ Manav Institute of Technology \& Management,Jevra -Hisar, Haryana,India \\ Rishi \\ Department of Computer \& Science Engineering \\ Manav Institute of Technology \& Management,Jevra-Hisar , Haryana,India
}

\begin{abstract}
The objective of the work is to reduce the high Peak to Average Power Ratio (PAPR) problem existing in OFDMA technology which highly impact QoS of signal. Performance analysis of different LTE techniques used as reduced PAPR OFDMA, SC-FDMA, MIMO) on the basis of QoS using BER and SNR parameters and to analyze all three techniques on the basis of their quality of service.
\end{abstract}

\section{INTRODUCTION}

In order to make its work worldwide a network of computers is organized. This organized collection of computers is called as Computer Network. A network of computers makes the easy share of resources such as data, information etc.

Different types of networks were raised with different features such as Local Area Network (LAN) having a restricted coverage area, mostly in organization and buildings, Metropolitan Area Network (MAN) covers the entire city and Wide Area Network (WAN) covers a huge geographical area such as a nation or even an entire world [8] [18].

- $\quad$ Wired Network

Networking is possible only through the use of wires which makes its use to a limited area and restrict its use in hill stations and through the oceans.

\section{- $\quad$ Wireless Network}

Wireless networking allows communication without the use of wires so troubles associated with setup and maintaining of wires totally detached out and the large area will be covered out.

Mobile communication mainly refers to a communication which does not depend on any physical connection between any two communication entities. It allows communication among moving entities.

3GPP LTE Network

LTE has been standardized by the 3rd Generation's Partnership Project (3GPP) as a part of 3GPP release. LTE mainly evolved with the growing needs of high data traffic both in magnitude and variety. The User may require fast uploading, downloading speed, online video gaming, video conferencing, etc. In order to meet this increasing demand of user during 2004, 3rd Generation Partnership Project (3GPP) [9] organized a workshop with various telecommunication industries such as Universal Mobile Telecommunication Unit (UMTS), Universal Terrestrial Radio Access network (UTRAN) and many other delights.

Performance Analysis of OFDMA in LTE

Farhood et al. [4] described that LTE uses OFDMA for downlink data transmission because of its different features such as better resistance to fading environments and high spectral efficiency, but still problems associated with this technique such as Inter Carrier Interference (ICI) and Inter Symbol Interference (ISI) which have to be removed. This paper described about the performance of an OFDMA system in LTE. After that, the main technologies must be explained, with probable improvements, their related challenges. They investigated the performance of OFDMA in LTE physical layer by taking into consideration diverse modulation schemes (BPSK, QPSK, 16QAM and 64QAM) on the basis of PAPR, power spectral density (PSD), BER and error probability. They concluded that the PAPR decreases for higher order modulation scheme and lower order 
modulation schemes (BPSK and QPSK) experience less BER at receiver that improve the system performance in terms of BER and SNR which show that the error probability increases as the order of modulation scheme increases.

\section{II. $\quad$ PROPOSED WORK}

STAGE1: Use of Clipping and filtering method in OFDMA. Clipping and Filtering is a significant method that is mainly used to reduce the High Peak to average Power (PAPR) Ratio problem associated with OFDMA.

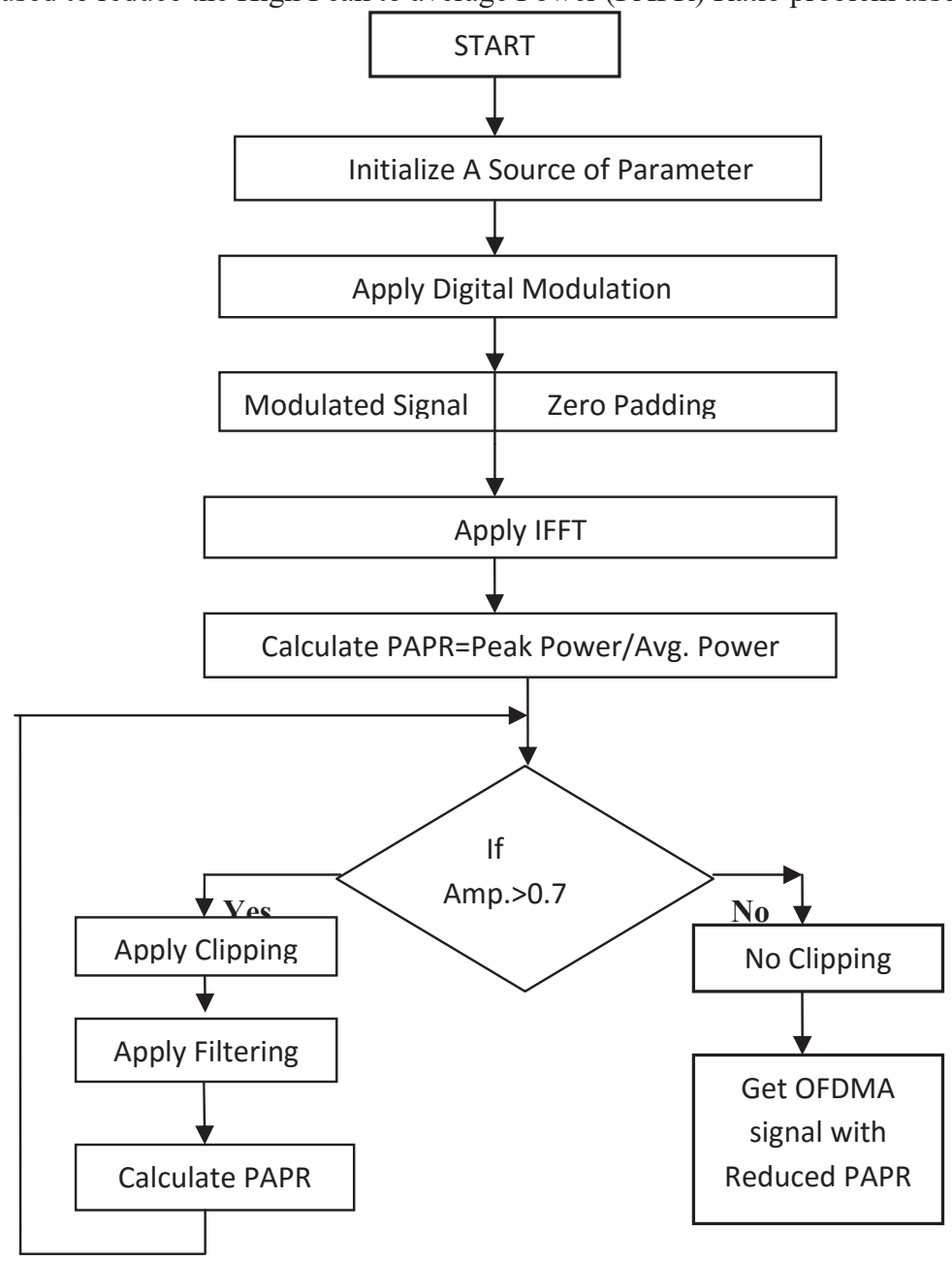

Figure 2.1: Flow Chart for Clipping and Filtering method used in OFDMA to reduce PAPR

STAGE2: In this stage we analysis the LTE Techniques on the basis of QoS. For this, We choose SNR and BER as a QoS parameter. These parameters are described in the next chapter. The technique we analyzed are reduced PAPR OFDMA, SC-FDMA and MIMO. In the analysis result, we take the different modulation scheme. Stepwise detailed is given below: 


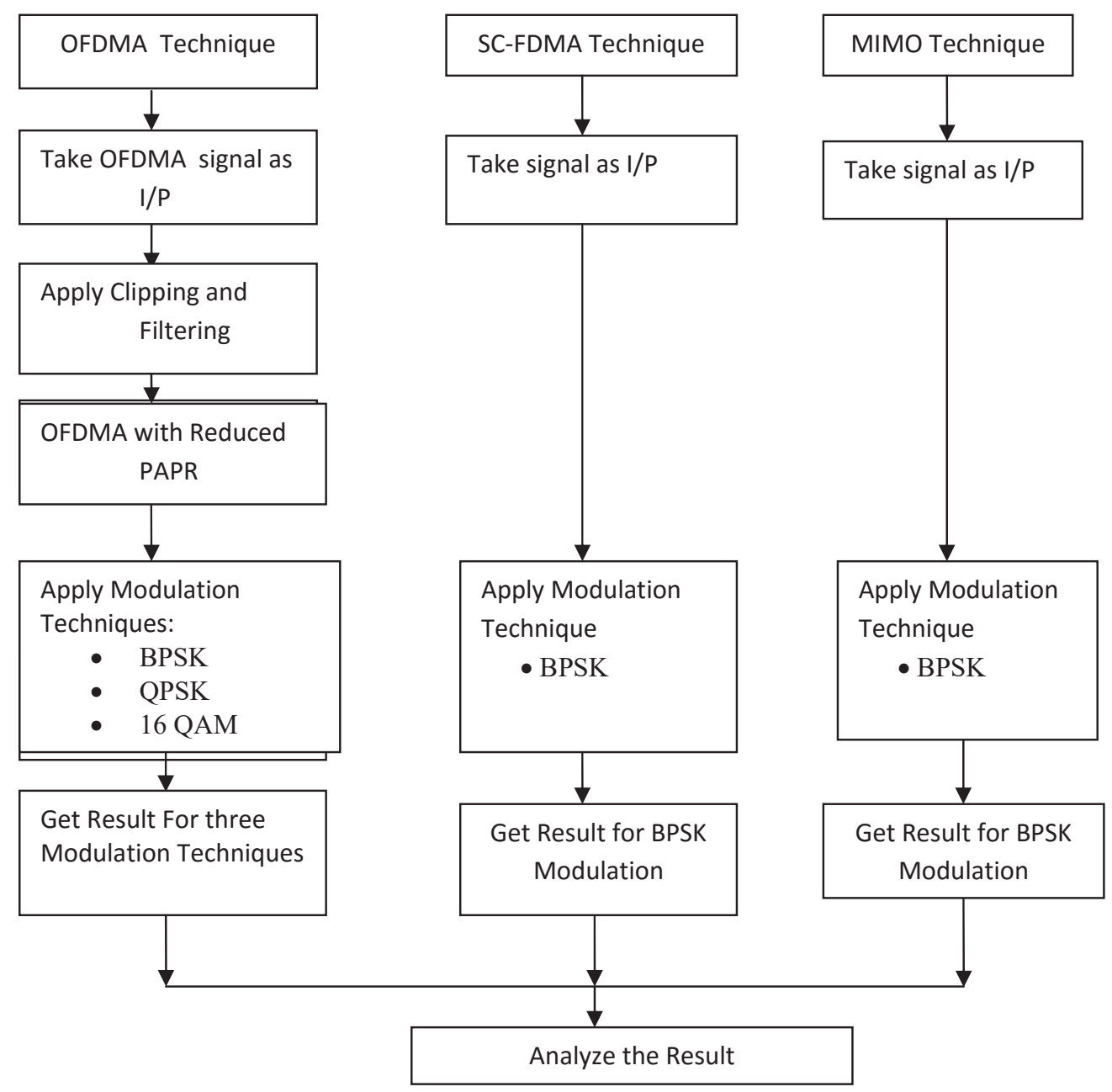

Figure 2.2: Flow Chart for Performance Analysis

III. RESULT AND ANALYSIS

The result of reduced PAPR OFDMA, SC-FDMA, and MIMO have been discussed and analyzed. Their performance in the MATLAB scenario in terms of different performance metrics i.e. SNR and BER has been described in the previous chapter for performance analysis.

Provides the result of OFDMA signal obtained after clipping and filtering method. Normal OFDMA indicates high peak to average power ratio. After we apply Clipping and filtering method we obtained a signal with small PAPR value as compared to the original 


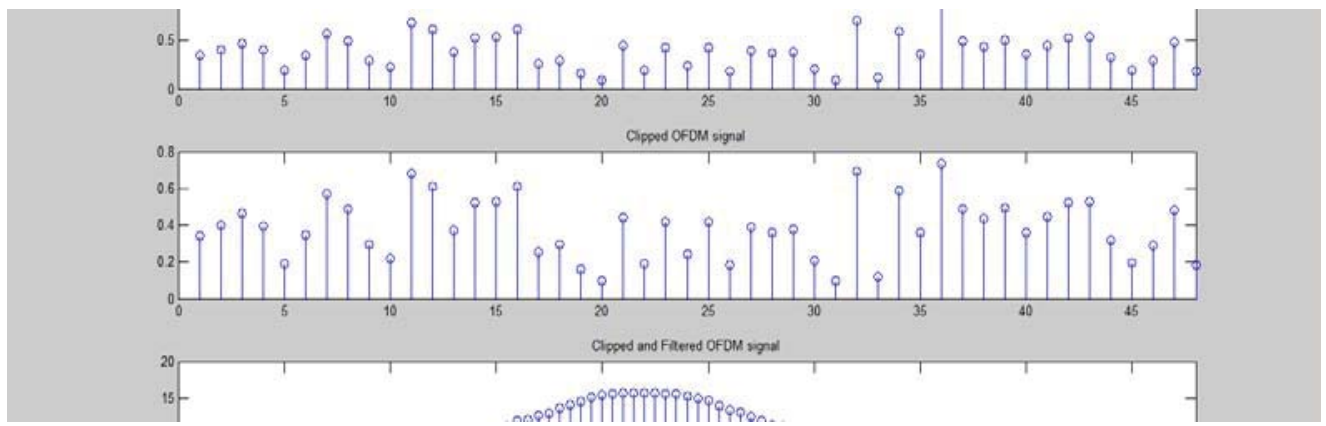

Fig 3.1 OFDMA signal after clipping and filtering method

The analysis of the three modulation techniques QPSK, 16 QAM, BPSK used in reduced PAPR OFDMA. All of these modulation techniques are analyzed on the basis of SNR and BER parameter. As there exists an inverse relation between SNR and BER.

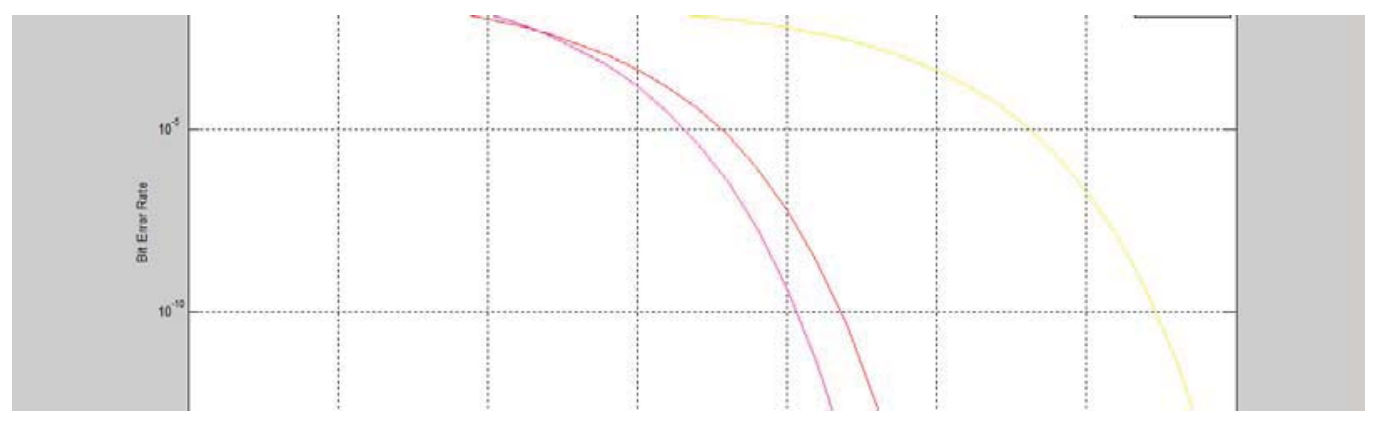

Fig 3.2 BER vs. SNR for OFDMA using QPSK, 16 QAM and BPSK

The result of MIMO signal. From our analysis from Figure 6.4 we found that BPSK modulation provides better results. We use BPSK modulation technique in MIMO and analyse its performance in MIMO on the basis of SNR and BER parameter. MIMO provides $10^{\wedge}-4.6 \mathrm{BER}$ value at $28 \mathrm{~dB}$ SNR.

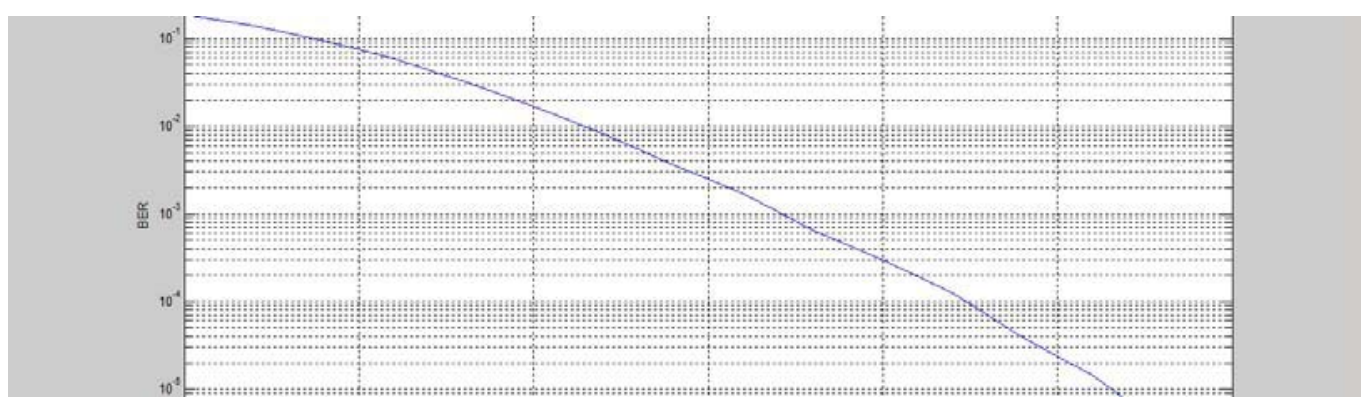

fig 3.3 BER vs SNR for MIMO 
The result of SC-FDMA signal. We found that BPSK modulation provides better results. We use BPSK modulation technique in SC-FDMA and analyze its performance in SC-FDMA on the basis of SNR and BER parameter. SCFDMA provides $10^{\wedge}-4.1 \mathrm{BER}$ value at $18 \mathrm{~dB}$ SNR and then constant.

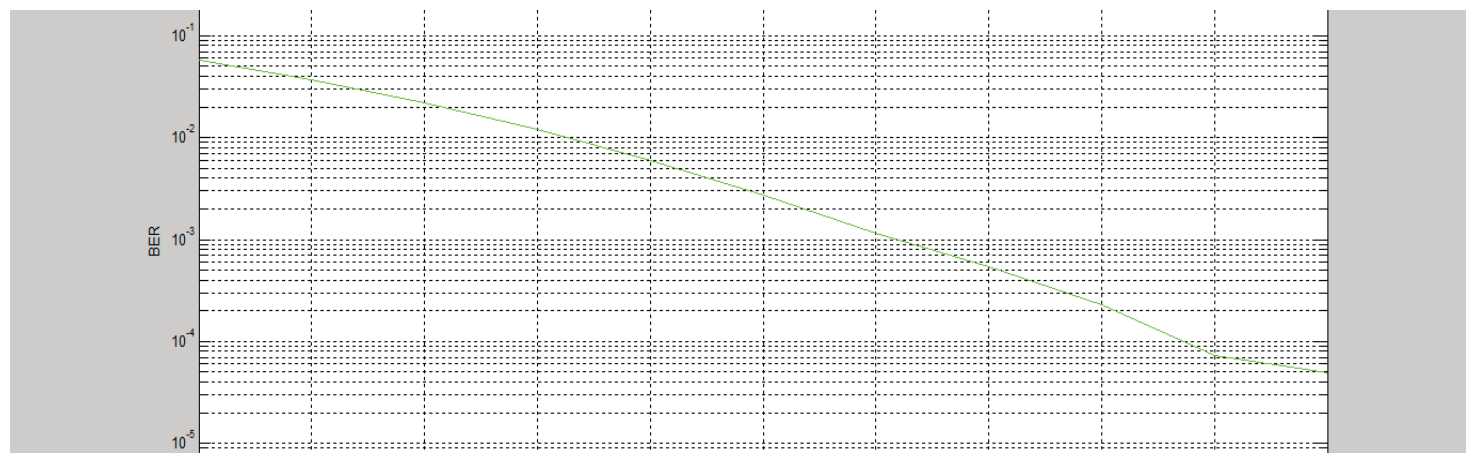

Fig 3.4 BER vs SNR for SCFDMA

The analysis of the three modulation techniques QPSK, 16 QAM, BPSK used in reduced PAPR OFDMA, BPSK modulation technique used in SC-FDMA and MIMO. All of these techniques are analyzed on the basis of SNR and BER . The signal which approach better BER value at low SNR provides better results.

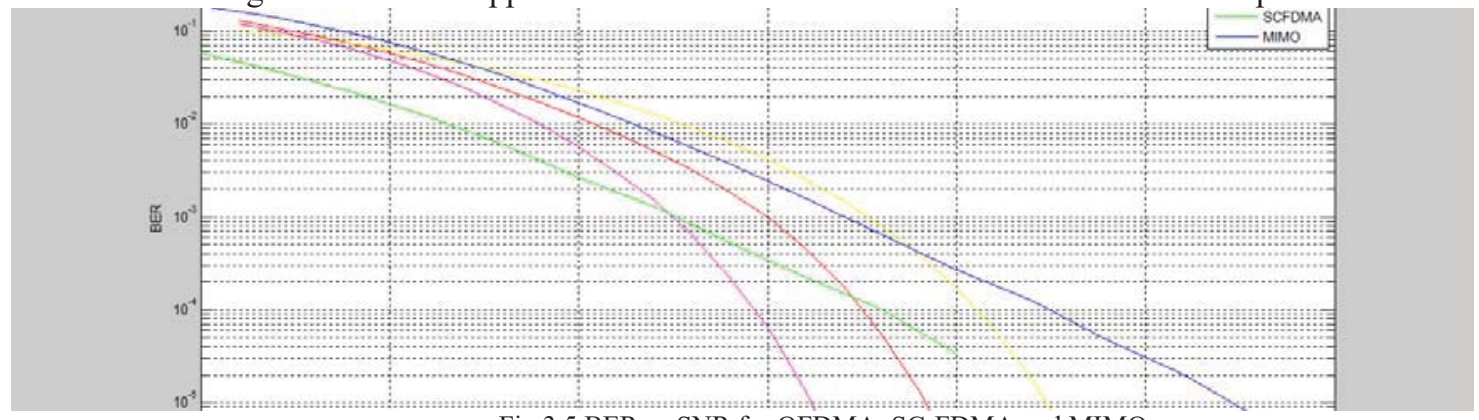

Fig 3.5 BER vs SNR for OFDMA, SC-FDMA and MIMO

\section{CONCLUSION AND FUTURE CONSIDERATIONS}

A better quality of service (QoS) is a very high increasing demand of Z Generation. 4G networks support a better quality of service mainly in terms of high data rate, large coverage area, low bit error rate, low cost but improvement is still going on. OFDMA is implemented using three modulation signals, i.e. QPSK, 16 QAM and BPSK. The high PAPR problem existing with OFDMA which highly impact the QoS of a signal is reduced using Clipping and Filtering method to make it able to provide better quality of service (QoS). Two techniques also implemented which compare with all three modulation techniques of reduced PAPR OFDMA. These techniques are MIMO and SCFDMA. In our research work BPSK (2) modulation technique added with MIMO and SC-FDMA that improve quality of signal. BER and SNR are important parameter that defines the quality of communication link. The decrease value of BER provides better result and show better quality of signal. The signal which approach better BER value at low SNR provides better result in terms of high quality of service.

From result analysis it is concluded that BPSK having better BER at low value of SNR which is the result of reduced PAPR OFDMA signal. From result analysis it is concluded that in all the techniques used in LTE reduced PAPR OFDMA having better quality of service as compared to MIMO and SC-FDMA.

In proposed work, we improved the service quality of OFDMA by applying efficient PAPR reduction technique and then we analyze the Quality of reduced PAPR OFDMA, MIMO and SC-FDMA on the basis of BER and SNR. The proposed work is effective but the work can be extended in future under following aspects: 
- The work can be improved by using another method for improving the quality of service of OFDMA for PAPR reduction such as Peak windowing, SLM technique etc.

- The work can be improved by using other modulation techniques.

- We can also calculate the performance on the basis of other parameters such as PAPR (Peak to Average Power Ratio), Congestion, PSD (Power Spectral Density) etc.

\section{REFERENCES}

[1] Sehrawat, M., Singh, R. and Sharma, P., "Performance Comparison Between OFDMA and SC-FDMA using Rayleing Fading Channel," International Journal of Advanced Technology in Engineering and Science (IJATES), Vol. 03, No. 01, March 2015.

[2] Sharmal, S. and Kumar, P., "Survey on PAPR Reduction Techniques in OFDM System," International Journal of Advanced Research in Computer and Communication Engineering (IJARCCE), Vol. 4, No. 6, June 2015.

[3] Jaiswal, S., Kumar, A. and Neha., "A Comphrensive Study of Development of Wireless Communication Network: from 1G to 5G," International Journal of Engineering and Computer Science (IJECS), Vol. 3, No. 05, pp. 6053-6056, May 2014.

[4] Farhood, A.D. and Agarwal, N., "Performance Analysis of OFDMA in LTE," International Journal of Current Engineering and Technology (IJCET), Vol. 4, No. 3, pp. 1614-1619, June 2014.

[5] Bhatia, V. and Gupta, J., "Minimization of Peak to Average Power Ratio in an OFDM System by using elective Mapping Technique," International Journal of Engineering Research \& Technology (IJERT), Vol.3, No. 7, July 2014.

[6] Deshar, A. and Agarwal, N., "Performance Analysis of OFDMA in LTE," International Journal of Current Engineering and Technology (IJCET), Vol. 4, No. 3, pp. 1614-1619, June 2014.

[7] Rao, A. and Srinivas, S., "A New BER and SNR Calculations for MIMO System" International Journal of Inventive Engineering and Sciences (IJIES), Vol. 2, No. 8, July 2014 